\title{
Tumor Specific Peptide
}

National Cancer Institute

\section{Source}

National Cancer Institute. Tumor Specific Peptide. NCI Thesaurus. Code C1906.

Protein fragments that are derived specifically from tumor cells and presented to T cells by Major Histocompatibility Complex (MHC) molecules expressed on antigen presenting cells or even on the tumors themselves. Often used alone or as conjugates with other molecules as experimental tumor vaccines. (NCI/OSP) 